\title{
Pseudo-pseudomyxoma peritonei from peritoneal sarcomatosis
}

\author{
Shuja Ahmed ${ }^{1}$, Ling Guo ${ }^{2}$, Shadi A. Qasem ${ }^{2}$, Edward A. Levine ${ }^{1}$ \\ 1. Surgical Oncology Service, Department of General Surgery, Wake Forest University School of Medicine, Winston Salem, \\ NC, USA. 2. Department of Pathology, Wake Forest University School of Medicine, Winston Salem, NC, USA.
}

Correspondence: Edward A. Levine, MD. Address: Surgical Oncology Service, Medical Center Blvd, Winston-Salem, North Carolina, USA. E-mail: elevine@wakehealth.edu

Received: February 12, 2015

Accepted: April 12, 2015

Online Published: June 3, 2015

DOI : $10.5430 /$ crcp.v2n4p14

URL: http://dx.doi.org/10.5430/crcp.v2n4p14

\section{Abstract}

Background: Pseudomyxoma peritonei (PMP) is a rare clinical entity of mucinous ascites, most commonly associated with appendiceal mucinous neoplasms. Cytoreductive surgery (CRS) and hyperthermic intraperitoneal chemotherapy (HIPEC) remains the current standard of care for PMP. Peritoneal sarcomatosis (PS) is an exceptionally rare disease with a poor prognosis. PMP associated with PS has not been previously described. The role of cytoreductive surgery and hyperthermic intraperitoneal chemotherapy for PS with or without PMP is not well-defined. PS manifesting like PMP has not been previously described.

Case presentation: A 74-year-old patient with several weeks history of vague abdominal pain and increased abdominal girth was referred to our facility after incidental finding of PMP during laparoscopic inguinal hernia repair. After complete work-up, he was advised to undergo CRS/HIPEC. Intra-operatively, he was noted to have extensive mucinous ascites and underwent aggressive CRS and HIPEC

Result: Final pathology revealed myxoid liposarcoma with associated intraperitoneal mucin dissemination, which was confirmed with cytogenetic analysis.

Conclusion: We describe a rare case of pseudomyxoma peritonei originating from an intra-abdominal sarcoma. We propose the term of "pseudo-pseudomyxoma peritonei” to appropriately describe this unusual clinical event.

\section{Key words}

Pseudomyxoma peritonei, Peritoneal sarcomatosis, Cytoreductive surgery

\section{Introduction}

Pseudomyxoma peritonei (PMP) refers to the slow, progressive accumulation of intra-abdominal mucin eventually leading to clinical ascites. Appendiceal mucinous tumors are the most common cause of PMP with epithelial ovarian tumors being the other common site of origin associated with PMP. Patients complain of abdominal distension with or without weight loss. Over time, however, worsening mucinous ascites leads to mechanical and functional bowel obstruction. If left untreated, the natural progression is death secondary to obstruction or cachexia. Cytoreductive surgery (CRS) and hyperthermic intraperitoneal chemotherapy (HIPEC) is associated with a survival benefit as well as long-term disease-free survival for patients with PMP ${ }^{[1]}$. 
Peritoneal dissemination of sarcoma or "sarcomatosis" results from intra-abdominal spread of intraperitoneal or retroperitoneal sarcoma. It is an aggressive disease associated with high recurrence and poor prognosis. Despite some similarities to PMP in its presentation, sarcomatosis is associated with a much higher recurrence and significantly worse prognosis. Further, there is no defined role of CRS/HIPEC for peritoneal sarcomatosis (PS) ${ }^{[2]}$.

We describe a unique case of a 74-year-old patient who presented with mucinous ascites secondary to PS. We propose the term pseudo-pseudomyxoma peritonei to describe this unique entity.

\section{Case presentation}

A 74-year-old man with past medical history of hypertension and coronary artery disease was referred to our clinic for evaluation of suspected mucinous peritoneal carcinomatosis. He had initially presented to his primary care physician a few weeks earlier with complaints of vague abdominal pain and increased "abdominal girth" over several months. He denied any weight loss, changes in appetite or pattern of bowel movements. On physical examination, he was noted to have bilateral inguinal hernias for which he underwent laparoscopic repair using the transabdominal pre-peritoneal technique. Intra-operatively, however, he was found to have peritoneal nodules with mucinous material in the peritoneal space. Biopsy of one of the peritoneal nodules revealed paucicellular mucoid material suggestive of mucinous neoplasm.

Upon presentation to our facility, his examination was significant for distended abdomen with dullness on percussion but no palpable mass. Prior staging work-up included a CT scan of abdomen/pelvis which demonstrated peritoneal dissemination of tumor involving mainly the right colon including the hepatic flexure and porta hepatis (see Figures 1, 2). No discrete colonic or appendiceal masses were noted. On full body PET/CT scan, these implants showed mild to moderate fluorodeoxyglucose $\left({ }^{18} \mathrm{FDG}\right.$ ) uptake. Laboratory tests were normal including CEA level of $0.4 \mathrm{ng} / \mathrm{ml}$ (normal value $<2.5 \mathrm{ng} / \mathrm{ml})$.

Figure 1. PET/CT scan demonstrating FDG uptake by peritoneal carcinomatosis involving the right colon

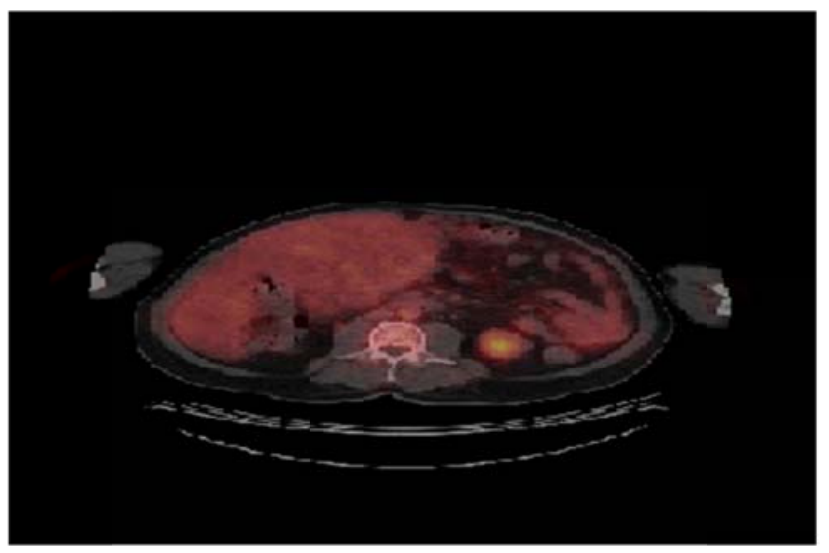

We proceeded with CRS/HIPEC with the presumptive diagnosis of appendiceal mucinous neoplasm. Intra-operatively, he was noted to have extensive peritoneal disease with mucinous material dispersed throughout the peritoneum (see Figure 3). The peritoneal carcinomatosis index score - a quantitative measure of the extent of peritoneal metastases - was 30, indicative of high tumor burden ${ }^{[3]}$. Extensive cytoreduction was performed including right hemicolectomy, omentectomy, cholecystectomy, partial gastrectomy with Billroth II reconstruction, bilateral diaphragmatic peritoneal stripping with right diaphragmatic resection and thoracostomy tube placement as well as debridement of tumor around the porta hepatis. After CC-1 (or R2a) resection, his abdomen was then perfused with oxaliplatin $\left(200 \mathrm{mg} / \mathrm{m}^{2}\right)$ at $40^{\circ} \mathrm{C}$ for one hour ${ }^{[4]}$.

The patient had a complicated post-operative course. After a protracted hospital stay, he was discharged to a nursing home, where he recovered for a month prior to returning home. On a recent six-month follow-up, he was noted to be doing well with no evidence of relapse. 
Figure 2. CT scan image demonstrating mucinous ascites or pseudomyxoma peritonei involving the porta hepatis
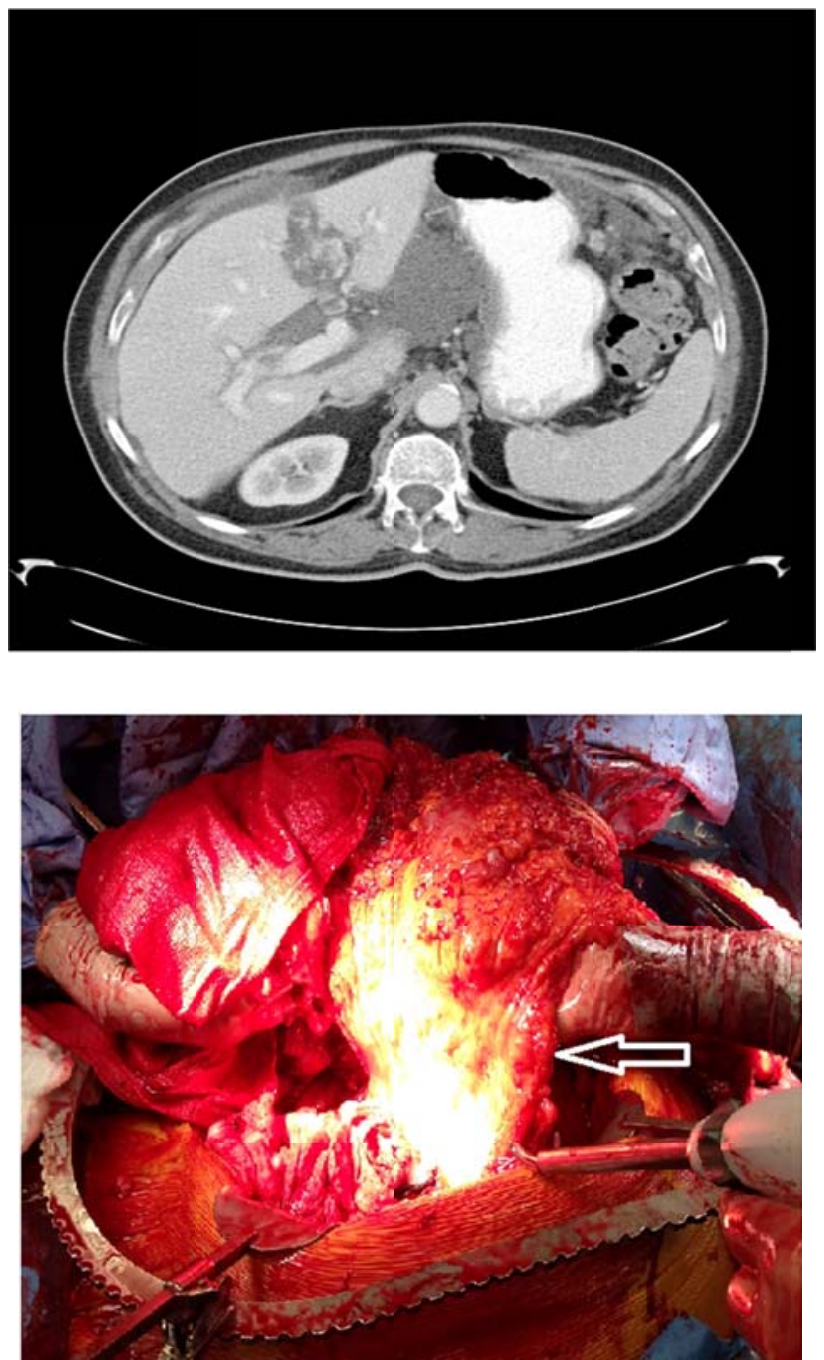

Figure 3. Intra-operative finding of mucin with extensive peritoneal surface disease (arrow)

Final pathology revealed myxoid liposarcoma (see Figure 4), with peritoneal dissemination and mucinous ascites. To further characterize the lesion, cytogenetic analysis for $\mathrm{C} /$ Enhancing binding protein homologous protein (CHOP) gene rearrangements at 12q13 was performed which revealed the gene to be disrupted, consistent with myxoid liposarcoma ${ }^{[5]}$.

Figure 4. Sections show a neoplasm composed of numerous vacuolated lipoblasts (black arrows) and capillary-type vasculature (white arrows). Notice the pale myxoid background. Scattered mature adipocytes are also noted (Hematoxylin and eosin, 20× magnification).

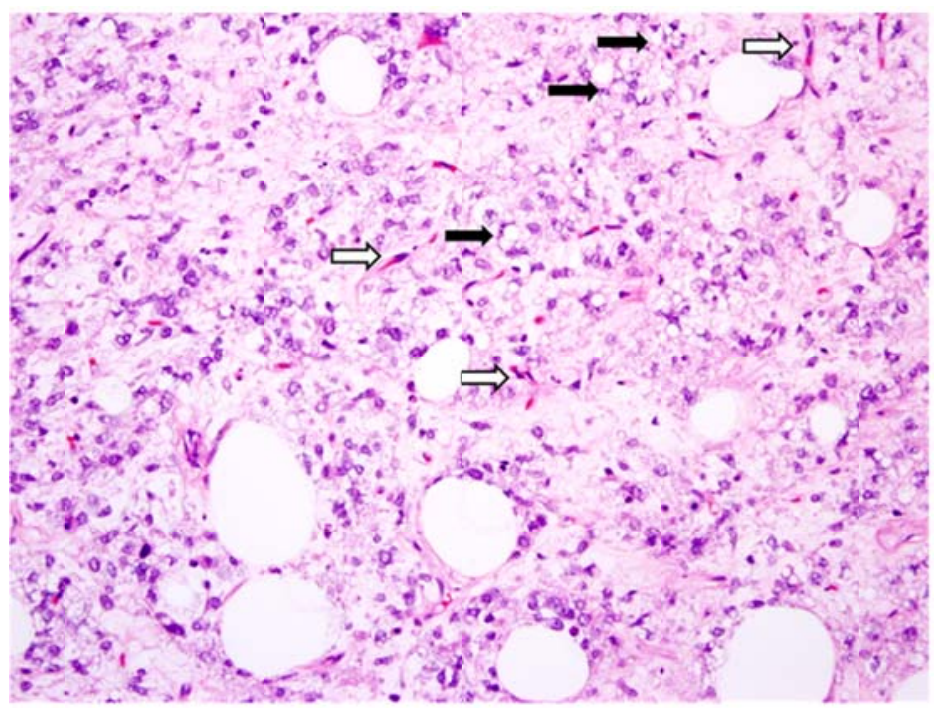




\section{Discussion}

With an approximate incidence of one patient per million ${ }^{[6]}$, PMP is a rare disease and remains poorly understood despite being first described in 1842 by Karl Rokitansky ${ }^{[7]}$. It is a clinical entity associated with progressive intra-abdominal mucin deposition secondary to many potential tumors usually of epithelial origin. Appendiceal tumors are the most common cause of PMP. Other causes of PMP include primary epithelial ovarian tumor and mucinous adenocarcinoma of the colon. PMP usually presents after the age of 40 and is slightly more common in women ${ }^{[8]}$.

The development of PMP is a slow process that evolves over several years. Its pathogenesis is best documented for appendiceal tumor and involves neoplastic transformation of goblet cell with production of large amounts of mucin resulting in a mucocele. Progressive secretion of mucin eventually results in rupture of the mucocele leading to PMP ${ }^{[9]}$. Intraperitoneal deposition of the goblet cells leads to accumulation of extra-cellular mucin, which is subsequently responsible for the morbidity and mortality associated with PMP.

Mucins are a diverse family of high molecular weight, heavily glycosylated proteins secreted by specialized epithelial cells of mucosal surfaces throughout the body. Mucin is easily identifiable on routine hematoxylin and eosin and can be highlighted by various histochemical stains. Normally, intraluminal mucin facilitates the transit of intestinal contents. However, progressive accumulation of intra-peritoneal mucin causes symptoms of abdominal distension with accompanying increased intra-abdominal pressure, which eventually leads to bowel obstruction.

Traditionally, epithelial tumors are known to produce mucin. However, mesenchymal tumors may also contain mucin, which is morphologically similar but structurally different from epithelial mucin ${ }^{[10]}$.

The therapeutic benefit of cytoreductive surgery and HIPEC for PMP has long been recognized. Five-year survival rates range between $66 \%-97 \%{ }^{[1]}$. Our institution has previously published outcomes for 472 patients with PMP from appendiceal primary with 10-year survival of approximately 30\%. Performance status, histologic grade, and resection status were independent predictors of survival ${ }^{[11]}$.

PS is the intra-abdominal dissemination of sarcoma. It is a rare event occurring usually with recurrent disease likely from tumor spillage during initial resection of the intra-abdominal sarcoma. PS carries a dismal overall prognosis due to the inherent resistance of most sarcomas to chemotherapy and radiation. Additionally, CRS/HIPEC is not consistently associated with significant improvement in survival for PS, particularly in high-grade disease, due to the high propensity for hematogenous dissemination ${ }^{[2]}$.

Naiki et al. ${ }^{[12]}$ have previously reported a case of retroperitoneal mucinous de-differentiated liposaroma. Our case, however, is unique in that the diagnosis of sarcomatosis with no prior history of intra-abdominal sarcoma and the associated PMP has, to our knowledge, never been reported before.

Despite the conflicting data for CRS/HIPEC in the setting of PMP versus PS, we believe our patient will gain survival benefit from removal of his mucinous ascites. However, he remains at high risk for recurrence due to the nature of primary tumor and incomplete resection status (R2a or CC-1). Once he gains full recovery, we will consult medical oncology for consideration of systemic chemotherapy. He is on aspirin for his coronary artery disease, which he was advised to continue as anti-inflammatory drugs can potentially reduce mucin secretion in PMP ${ }^{[13]}$.

\section{Conclusion}

We present a case of pseudomyxoma peritonei from a non-epithelial neoplasm. We believe the term "pseudopseudomyxoma peritonei” best describes this unusual clinical event. This diagnosis should be considered when a mucinous tumor of non-epithelial origin is found in a biopsy specimen. 


\section{References}

[1] Smeenk RM, Verwaal VJ, Antonini N, et al. Survival analysis of pseudomyxoma peritonei patients treated by cytoreductive surgery and hyperthermic intraperitoneal chemotherapy. Ann Surg. 2007 Jan; 245(1): 104-9. PMid: 17197972. http://dx.doi.org/10.1097/01.sla.0000231705.40081.1a

[2] Randle RW, Swett KR, Shen P, et al. Cytoreductive surgery with hyperthermic intraperitoneal chemotherapy in peritoneal sarcomatosis. Am Surg. 2013 June; 79(6): 620-624. PMid: 23711273.

[3] Jacquet P, Sugarbaker PH. Clinical research methodologies in diagnosis and staging of patients with peritoneal carcinomatosis. Cancer Treat Res. 1996; 82: 359-74. http://dx.doi.org/10.1007/978-1-4613-1247-5_23

[4] Harmon RL, Sugarbaker PH. Prognostic indicators in peritoneal carcinomatosis from gastrointestinal cancer. Int Semin Surg Oncol. 2005; 2: 3. http://dx.doi.org/10.1186/1477-7800-2-3

[5] Suzuki K, Matsui Y, Endo K, et al. Myxoid liposarcoma with EWS-CHOP type 1 fusion gene. Anticancer Res. 2010 Nov; 30(11): 4679-83. PMid: 21115923.

[6] Smeenk RM, van Velthuysen ML, Verwaal VJ, et al. Appendiceal neoplasms and pseudomyxoma peritonei: a population based study. Eur J Surg Oncol. 2008 Feb; 34(2): 196-201. PMid: 17524597. http://dx.doi.org/10.1016/j.ejso.2007.04.002

[7] Weaver CH. Mucocele of the appendix with pseudomucinous degeneration. Am J Surg. 1937; 36: 523-526. http://dx.doi.org/10.1016/S0002-9610(37)90763-3

[8] Li C, Kanthan R, Kanthan SC. Pseudomyxoma peritonei - a revisit: report of 2 cases and literature review. World J Surg Oncol. 2006; 4: 60. http://dx.doi.org/10.1186/1477-7819-4-60

[9] Amini A, Masoumi-Moghaddam S, Ehteda A, et al. Secreted mucins in pseudomyxoma peritonei: pathophysiological significance and potential therapeutic prospects. Orphanet J Rare Dis. 2014 May 5; 9: 71. http://dx.doi.org/10.1186/1750-1172-9-71

[10] Graadt van Roggen JF, Hogendoorn PC, Fletcher CD. Myxoid tumours of soft tissue. Histopathology. 1999 Oct; $35(4)$ : $291-312$. PMid: 10564384. http://dx.doi.org/10.1046/j.1365-2559.1999.00835.x

[11] Levine EA, Stewart JH, Shen P, et al. Intraperitoneal chemotherapy for peritoneal surface malignancy: experience with 1,000 patients. J Am Coll Surg. 2014 Apr; 218(4): 573-85. http://dx.doi.org/10.1016/j.jamcollsurg.2013.12. 013

[12] Naiki T, Hamamoto S, Kawai N, et al. Giant Retroperitoneal Mucinous Tumor Supportively Diagnosed as a Dedifferentiated Liposarcoma by Fluorescence In Situ Hybridization of MDM2 Gene. ISRN Urol. 2011; 2011: 261735. http://dx.doi.org/10.5402/2011/261735

[13] Choudry HA, Mavanur A, O'Malley ME, et al. Chronic anti-inflammatory drug therapy inhibits gel-forming mucin production in a murine xenograft model of human pseudomyxoma peritonei. Ann Surg Oncol. 2012 May; 19(5): 1402-9.

http://dx.doi.org/10.1245/s10434-012-2242-5 